\title{
Decision speed for the choosing of the larger or the smaller of two digits
}

BENJAMIN A. FAIRBANK, JR.,' and JACK CAPEHART, University of Arizona, Tucson, Ariz. 85721

$A$ recent published report suggests that numbers may be processed internally as analog magnitudes when $S s$ are required to select the larger of two digits. If this is the case then it is suggested that Ss should be able to select larger digits more quickly than smaller digits. Experimental investigation confirms that the choosing of larger digits is faster than the choosing of smaller digits.

In 1967 Moyer \& Landauer presented evidence which implies that the judgmental process involved in selecting the larger (in numerical value) of two digits is analogous to the process involved in selecting the greater of two physical magnitudes. When an $\mathrm{S}$ selects the larger of two digits, Moyer and Landauer report, the time required for the selection varies negatively with the difference between the digits. This finding has been successfully replicated (Fairbank, 1969), but nevertheless the contention that numerical inequalities are judged in essentially the same way that physical inequalities are judged is somewhat difficult to accept. Intuitively the judgment that 7 is greater than 3 would seem to be more symbolic or cognitive than the judgment that $76 \mathrm{~g}$ feels heavier than $58 \mathrm{~g}$. Accordingly, the authors attempted to derive further predictions from the assertion that digits are somehow processed as analog magnitudes. The prediction that Ss could select the larger of two digits more quickly than the smaller was arrived at on the basis of the following considerations.

Adaptation-level (AL) theory (Helson, 1964) implies that stimuli are judged with respect to the prevailing adaptation level of a given set of stimuli. That is, the prevailing $\mathrm{AL}$ acts as a frame of reference with respect to which categorizing judgments are made. Typically, the AL is decentered, i.e., it is below the arithmetic mean of the stimulus set.

Helson (1964) asserts that psychological contrast is a function of the distance from the prevailing $A L$ to the stimulus being judged. An implication of the decentered $A L$ is that the stimulus furthest above $\mathrm{AL}$ has greater contrast than the stimulus furthest below $A L$ because with a decentered $A L$ the larger member of a stimulus pair is a greater distance from the AL.

It is assumed for this study that decision time is an indicator of psychological contrast, as Capehart \& Pease (1968) demonstrated in a lifted-weight experiment. In other words, the more distant a stimulus is from the prevailing $\mathrm{AL}$, the more rapidly may that stimulus be categorized. Therefore, if the AL for a given pair of digits is decentered toward the smaller digit, the decision time should be shorter for the selection of the larger digit than for the selection of the smaller. The present experiment was designed to test this possibility.

\section{METHOD}

The complete list of 72 possible nonidentical pairs of digits was randomized eight times. Two complete random orders of these digits were typed on each of four spirit duplicating masters, yielding 144 pairs of digits on each master. Twenty-four copies of each master were made, and 24 booklets of four pages each were made up. Each booklet had the four pages arranged in a different order, thus all possible orders of pages were used. On each page, six columns of 24 pairs appeared, the digits of each pair were typed three spaces apart, adjacent pairs were separated by eight spaces horizontally and double spacing vertically. Ss had the task of crossing out the larger or the smaller digit, depending on instructions, of each pair as quickly as possible, and were timed for each page. Ss were given a 1 -min rest between pages.

Half of the Ss crossed out the larger digits on the first page, the smaller digits of the next two pages, and the larger ones on the last page. The other half started by crossing out the smaller digits, then completed two pages of larger digits, and finally completed one page of smaller ones. Thus there was virtually no chance that the order of administration affected the outcome of the experiment.

Twenty-four volunteer university student Ss took part, each working alone so that $\mathrm{E}$ could time the pages accurately. The dependent variable was the time required to complete each individual page as measured by a stopwatch.

\section{RESULTS}

The mean time per page for crossing out the larger digits was $89.8 \mathrm{sec}$, while the mean for crossing out the smaller digits was $93.3 \mathrm{sec}$ per page. Seventeen of the 24 Ss were faster crossing out the larger digits, and one showed no difference. The times required for the pages with the same instructions (e.g., "cross out the smaller digit") were combined to make a two-column by 24-row data table suitable for analysis with a Ss by Conditions analysis of variance. Such an analysis showed that the difference between selecting the larger and selecting the smaller digit was significant at the 0.0012 level $[F(1,23)=13.91]$. The average time difference of $3.5 \mathrm{sec}$ between the pages may also be interpreted as a difference of $24 \mathrm{msec}$ per pair, i.e., on the average, Ss were able to cross out the larger element of a pair 24 msec faster than they were able to cross out the smaller.

\section{DISCUSSION}

The experimental results support the hypothesis that $\mathrm{AL}$ theory is a concept relevant to the judging of numerical inequalities and thus lend credence to Moyer \& Landauer's (1967) suggestion that numerical inequalities are judged in a way analogous to the judging of physical inequalities.

An apparent decentering of the $\mathrm{AL}$ even when numbers rather than physical magnitudes are used as stimuli was found although not discussed by Parducci, Calfee, Marshall, \& Davidson (1960) in a report describing several experiments in which Ss had to estimate the means of large series of numbers. Fairbank (in press) has obtained preliminary data showing that Ss tend to underestimate the true mean when asked to estimate the midpoint between two two-digit numbers but are constrained from actually calculating the mean. Thus the assumption of a decentered $\mathrm{AL}$ appears defensible in the case of numerical stimuli.

\section{REFERENCES}

CAPEHART, J., \& PEASE, V. An application of adaptation-level theory to transposition responses in a conditional discrimination. Psychonomic Science, 1968, 10, 147-148.

FAIRBANK, B. A. JR. Experiments on the temporal aspects of number perception. 1969. (Doctoral dissertation, University of Arizona) Ann Arbor, Mich.: University Microfilms, in press.

HELSON, H. Adaptation level theory, an experimental and systematic approach to behavior. New York: Harper and Row, 1964.

MOYER, R. S., \& LANDAUER, T. K. Time required for judgments of numerical inequality. Nature, $1967,215,1519-1520$.

PARDUCCI, A., CALFEE, R. C., MARSHALL, L. M., \& DAVIDSON, L. P. Context effects in judgment: Adaptation level as a function of the mean, midpoint, and median of the stimuli. Joumal of Experimental Psychology, 1960, 60, 65-77.

\section{NOTES}

1. Present address: Institute for Perception RVO-TNO, Kampweg 5, Postbus 23, Soesterberg, The Netherlands.

2. This report is based on one part of Benjamin Fairbank's dissertation which was prepared while he was supported by a NASA predoctoral traineeship. National Science Foundation Grant GB 3955 to the University of Arizona helped to support this research. 